\title{
Molecular biomarkers in extrahepatic bile duct cancer patients undergoing chemoradiotherapy for gross residual disease after surgery
}

\author{
Hyeon Kang Koh, MD, Hae Jin Park, MD², Kyubo Kim, MD', \\ Eui Kyu Chie, MD¹, Hye Sook Min, MD³ Sung W. Ha, MD' \\ Departments of ${ }^{1}$ Radiation Oncology and ${ }^{3}$ Pathology, Seoul National University College of Medicine, Seoul; \\ ${ }^{2}$ Department of Radiation Oncology, Soonchunhyang University Hospital, Seoul, Korea
}

\begin{abstract}
Purpose: To analyze the outcomes of chemoradiotherapy for extrahepatic bile duct (EHBD) cancer patients who underwent R2 resection or bypass surgery and to identify prognostic factors affecting clinical outcomes, especially in terms of molecular biomarkers.

Materials and Methods: Medical records of 21 patients with EHBD cancer who underwent R2 resection or bypass surgery followed by chemoradiotherapy from May 2001 to June 2010 were retrospectively reviewed. All surgical specimens were reevaluated by immunohistochemical staining using phosphorylated protein kinase B (DAKT), CD24, matrix metalloproteinase 9 (MMP9), survivin, and $\beta$-catenin antibodies. The relationship between clinical outcomes and immunohistochemical results was investigated. Results: At a median follow-up of 20 months, the actuarial 2-year locoregional progression-free, distant metastasis-free and overall survival were $37 \%, 56 \%$, and 54\%, respectively. On univariate analysis using clinicopathologic factors, there was no significant prognostic factor. In the immunohistochemical staining, cytoplasmic staining, and nuclear staining of pAKT was positive in 10 and 6 patients, respectively. There were positive CD24 in 7 patients, MMP9 in 16 patients, survivin in 8 patients, and $\beta$-catenin in 3 patients. On univariate analysis, there was no significant value of immunohistochemical results for clinical outcomes.

Conclusion: There was no significant association between clinical outcomes of patients with EHBD cancer who received chemoradiotherapy after $\mathrm{R} 2$ resection or bypass surgery and pAKT, CD24, MMP9, survivin, and $\beta$-catenin. Future research is needed on a larger data set or with other molecular biomarkers.
\end{abstract}

Keywords: Extrahepatic bile duct cancer, Chemoradiotherapy, Immunohistochemistry, Molecular biomarker

\section{Introduction}

Extrahepatic bile duct (EHBD) cancer is a rare malignancy [1]. Complete surgical resection provides the only possibility of cure, but it could be considered to a limited number of patients with early stage disease [2]. Between $50 \%$ and $90 \%$ of patients with bile duct cancer present with locally unresectable disease [3]. It has been known that the outcome of EHBD cancer is dismal. The 5 -year survival rate is approximately $20 \%$ even after complete surgical resection [4]. Long term survivors cannot be expected after $\mathrm{R} 2$ resection or bypass surgery $[5,6]$.

To improve outcomes, several researchers have tried to find

Received 17 September 2012, Revised 17 October 2012, Accepted 22 October 2012.

Correspondence: Kyubo Kim, MD, Department of Radiation Oncology, Seoul National University College of Medicine, 103 Daehak-ro, Jongno-gu, Seoul 110-799, Korea. Tel: +82-2-2072-4019, Fax: +82-2-765-3317, E-mail: kyubokim@snu.ac.kr

(c) This is an Open Access article distributed under the terms of the Creative Commons Attribution Non-Commercial License (http://creativecommons.org/ licenses/by-nc/3.0/) which permits unrestricted non-commercial use, distribution, and reproduction in any medium, provided the original work is properly cited.

www.e-roj.org 
out prognostic factors for EHBD cancer [7-18]. Especially, some molecular biomarkers were published as prognostic factors [12-18]. However, most studies were based on surgical series and patient population was heterogeneous especially in terms of adjuvant therapy employed.

The aim of this study is to analyze the outcomes of chemoradiotherapy for EHBD cancer patients with gross residual disease after surgery and to identify prognostic factors affecting clinical outcomes, especially, in terms of immunohistochemical results of molecular biomarkers such as phosphorylated protein kinase B (pAKT), CD24, matrix metalloproteinase 9 (MMP9), survivin, and $\beta$-catenin.

\section{Materials and Methods}

\section{Patient population and treatment}

After institutional review board approval, surgical and pathologic reports were reviewed. From May 2001 to June 2010, 21 patients with EHBD cancer underwent chemoradiotherapy or radiotherapy alone after $\mathrm{R} 2$ resection or bypass surgery.

As for surgical procedures, 19 patients had R2 resection such as palliative partial hepatectomy, hilar resection, bile duct resection and pancreaticoduodenectomy. Two patients had palliative bypass surgery.

Most patients $(n=18)$ were treated with concurrent chemoradiotherapy. Concomitant 2 cycles of 5 -fluorouracil (5-FU) was given during radiotherapy. Among these patients, 15 patients continued maintenance chemotherapy after completion of chemoradiotherapy. Maintenance chemotherapy consisted of the median 5.5 cycles (range, 2 to 6 cycles) of 5 -FU and leucovorin in 12 patients, 6 cycles of 5-FU in 2 patients and 8 cycles of 5-FU and cisplatin in one patient. Three patients received radiotherapy alone. The median duration from surgery to start of radiotherapy was 47 days (range, 35 to 145 days). The median radiation dose was 50.4 Gy (range, 40 to $55.8 \mathrm{~Gy}$ ). Fraction size of each treatment was 1.8 or $2.0 \mathrm{~Gy}$. Sixteen patients received continuous course radiotherapy, while 5 patients received split course radiotherapy which consisted of a total of $40 \mathrm{~Gy}$ at 2 Gy per fraction with 2-week planned rest after $20 \mathrm{~Gy}$. Radiotherapy technique was 2-dimensional radiotherapy in 3 patients, 3-deminsional conformal radiotherapy in 16 patients and intensity modulated radiotherapy in 2 patients. The treatment volume encompassed gross residual disease, tumor bed and regional lymph nodes.

\section{Immunohistochemistry}

All 21 cases diagnosed as EHBD cancer were retrieved from the archives, which contained enough paraffin-embedded tissue for the study. After a review of the tumor sections of each case of cancer, one of the representative area of each donor block was punched out with a core of $4 \mathrm{~mm}$ diameter from formalin-fixed paraffin-embedded blocks and arranged in new recipient tissue microarray (TMA) blocks using a trephine apparatus (Superbiochips Laboratories, Seoul, Korea).

The TMA blocks were sectioned at 5- $\mu \mathrm{m}$ thickness, transferred by water flotation, and dried at room temperature. Immunohistochemistry was performed on a Bond Max autostainer (Leica Microsystems, Bannockburn, IL, USA) using Bond Polymer Detection kit (Leica Biosystems). Immunohistochemical staining using antibodies to PAKT (rabbit monoclonal antibody; 1:100; Epitomics Inc., Burlingame, CA, USA), CD24 (mouse monoclonal antibody; 1:200; Thermo Scientific Korea, Seoul, Korea), MMP9 (rabbit polyclonal antibody; 1:100; Neomarkers, Fremont, CA, USA), survivin (rabbit polyclonal antibody; 1 : 500; REtD Systems, Minneapolis, MN, USA), and $\beta$-catenin (mouse monoclonal antibody: 1:500; BD Bioscience, San Jose, CA, USA) was done on all 21 cases automatically according to the manufacturer's protocol based on the conventional streptavidin-biotin-peroxidase method.

\section{Immunohistochemical evaluation}

Reading of imunohistochemical results was performed by one pathologist. For PAKT expression analysis, staining of cytoplasm or nucleus was considered positive (Fig. 1A). For CD24, MMP9, and survivin expression analysis, staining of cytoplasm was considered positive (Fig. 1B-D). For $\beta$-catenin expression analysis, staining of nucleus was considered positive (Fig. 1E).

\section{Statistical analysis}

Locoregional progression was defined as progression in primary site and regional lymph nodes, and distant metastasis was defined as progression in outside of locoregional area by followup imaging studies. Locoregional progression-free survival (LRPFS), distant metastasis-free survival (DMFS), and overall survival (OS) were counted from the date of surgery to the date of each event and calculated by the Kaplan-Meier method. Twosided log-rank tests were used for the comparison of outcomes between groups. A p-value less than 0.05 in a two-sided test was regarded statistically significant. Data was analyzed using SPSS ver. 18.0 (SPSS Inc., Chicago, IL, USA). 

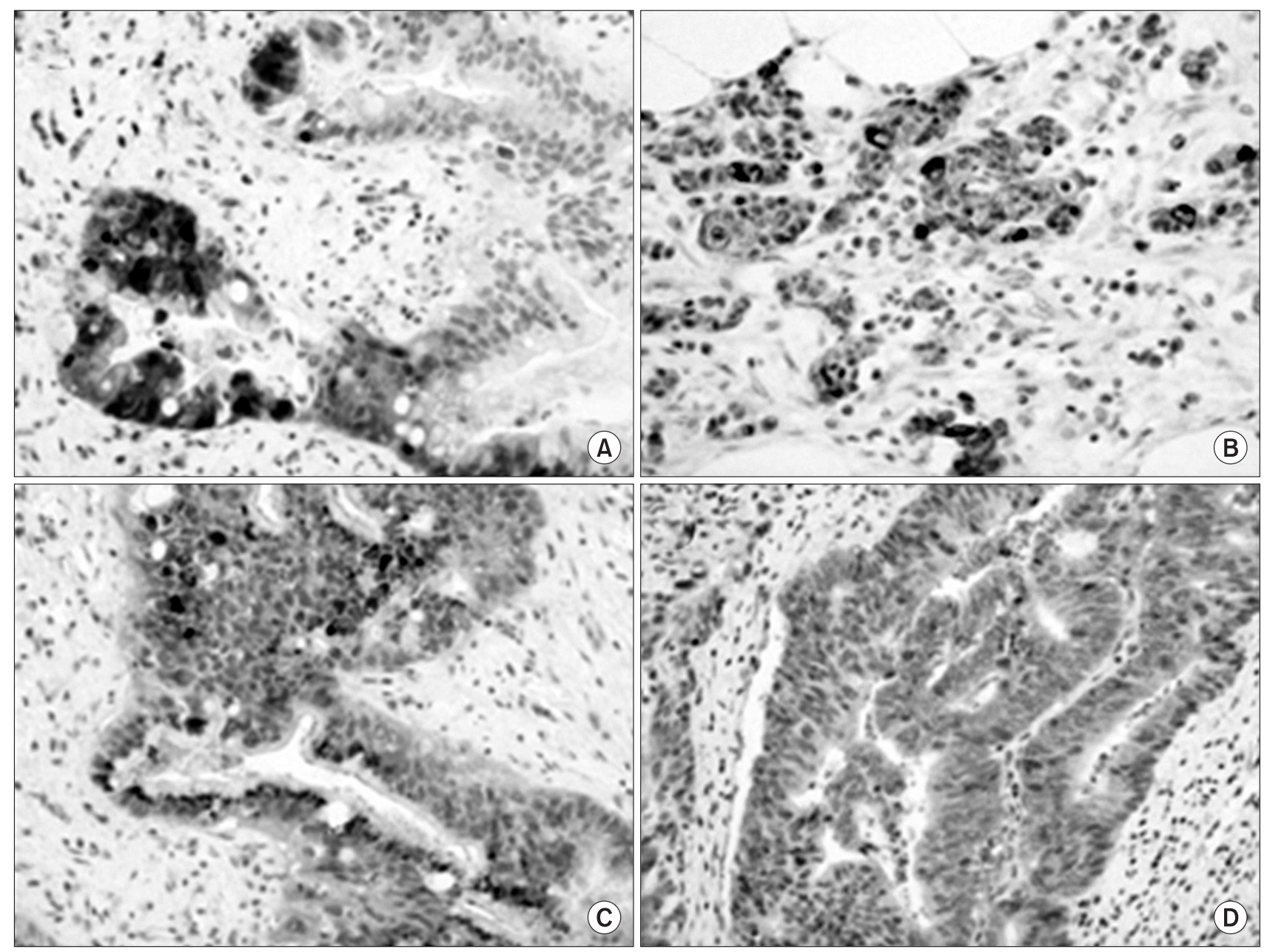

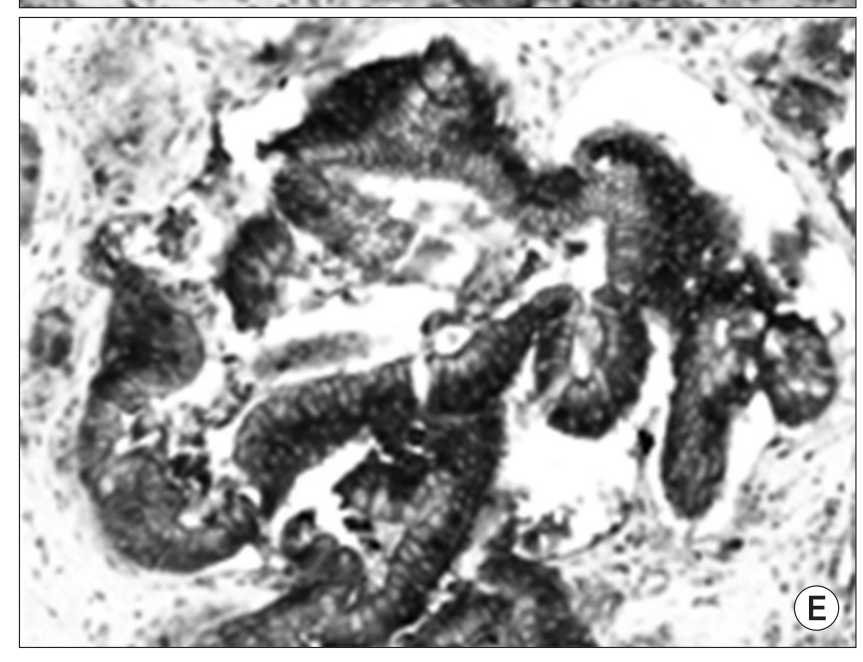

Results

\section{Patient characteristics}

The median age of patients at surgery was 62 years (range, 24 to 79 years). Nine patients were female, and 12 patients were
Fig. 1. Immunohistochemical staining of phosphorylated protein kinase B (pAKT) positive in nucleus and cytoplasm (A), CD24 positive in cytoplasm (B), matrix metalloproteinase 9 positive in cytoplasm (C), survivin positive in cytoplasm (D), and $\beta$-catenin positive in nucleus (E) (x400).

male. Most patients $(n=19)$ were of the Eastern Cooperative Oncology Group (ECOG) performance status 0 or 1. Tumor location was hilar in 16 patients (Klatskin tumor) and non-hilar (common bile duct cancer) in 5 patients. Eight patients had regional lymph node involvement. In terms of Klatskin tumor, 

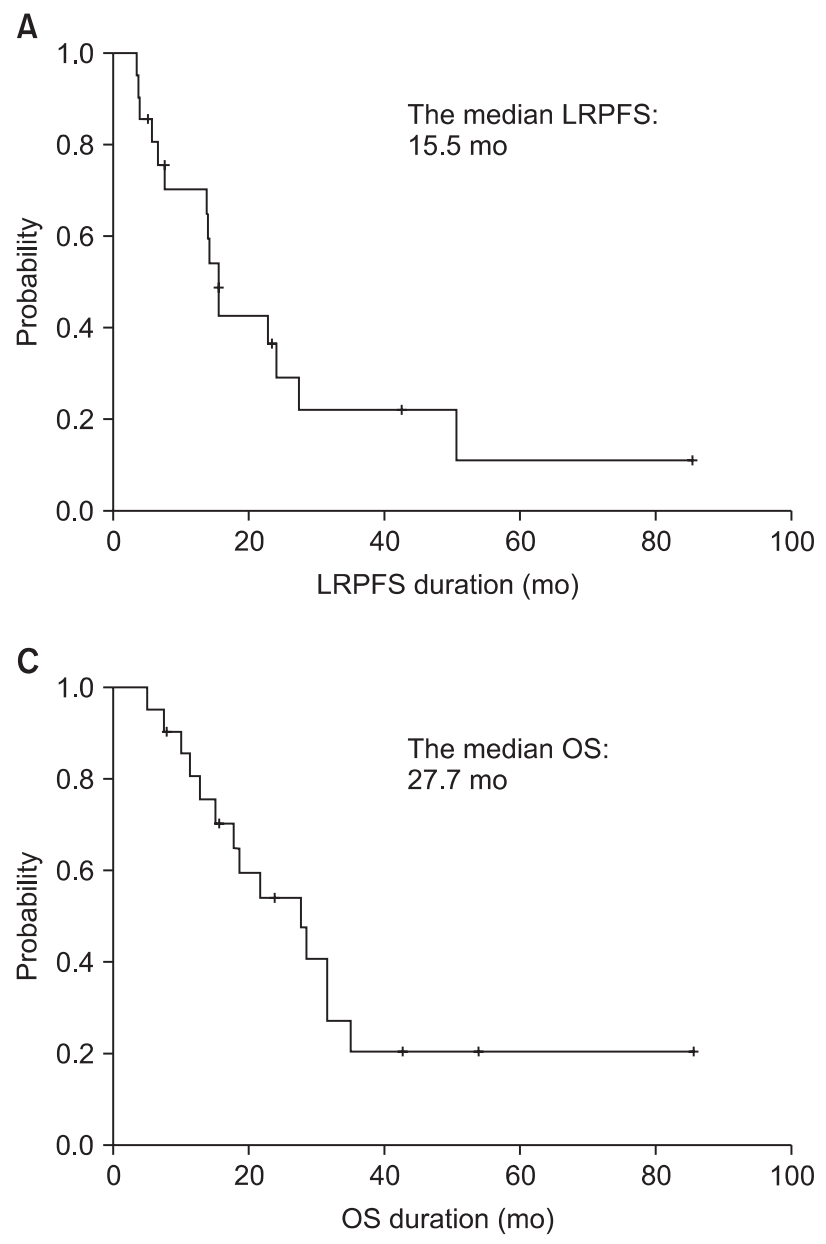

stage by the American Joint Committee on Cancer (AJCC) 7th edition was II in 4 patients, IIIA in 6 patients, IIIB in 5 patients, and IVA in one patient. T stage was T2 in 5 patients, T3 in 10 patients, and T4 in one patient. In terms of common bile duct cancer, stage was IB in 3 patients, II in one patient, and III in one patient. T stage was T2 in 4 patients and T4 in one patient.

\section{Treatment outcomes and prognostic factors}

The median follow-up duration was 20 months (range, 5 to 85 months). During follow-up, there were 15 cases of locoregional progression, 10 cases of distant metastases, and 14 cases of deaths. The actuarial 2-year LRPFS, DMFS, and OS rates were $37 \%, 56 \%$, and 54\%, respectively. The median LRPFS, DMFS, and OS were 15.5, 24.0, and 27.7 months, respectively (Fig. 2).

Age, gender, ECOG performance status, tumor location, nodal involvement, preoperative CA 19-9 level, type of operation and treatment were analyzed in univariate analysis for evaluation of prognostic significance of clinicopathologic features (Table 1). There was no significant prognostic factor on univariate analysis.

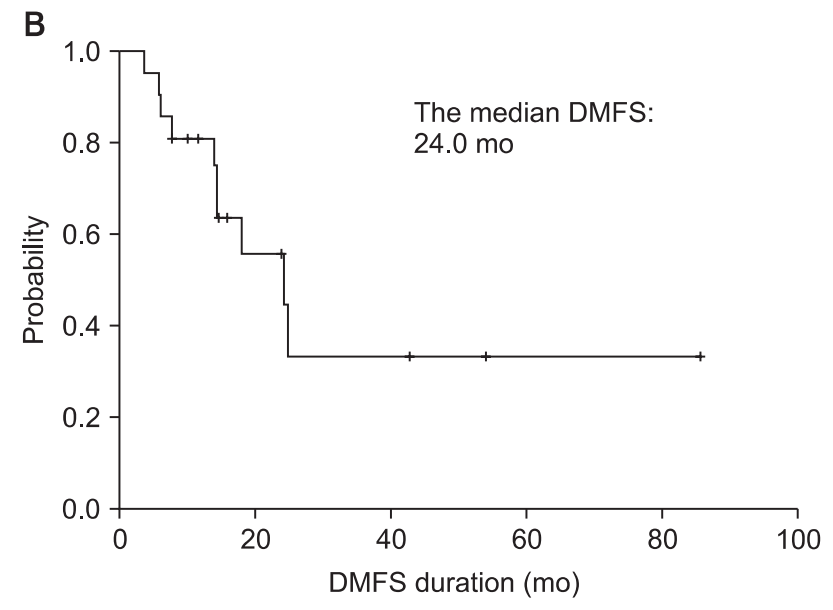

Fig. 2. Survival curves of (A) locoregional progression-free survival (LRPFS), (B) distant metastasis-free survival (DMFS), and (C) overall survival (OS).

\section{Immunohistochemical results}

Ten patients had positive tumors for pAKT cytoplasmic staining, but 6 patients had positive tumors for pAKT nuclear staining. As for CD24, 14 patients had negative results. When MMP9 was evaluated, there were 16 patients with positive cytoplasmic staining. As for survivin, there were 8 patients with cytoplasmic staining. According to the expression of $\beta$-catenin, 3 patients were positive in nuclear staining. Table 2 shows univariate analysis of immunohistochemical results. There was also no significant prognostic factor.

\section{Discussion and Conclusion}

In the present study, the expressions of pAKT, CD24, MMP9, survivin, and $\beta$-catenin were not associated with the prognosis of patients with EHBD cancer who received chemoradiotherapy after $\mathrm{R} 2$ resection or bypass surgery.

It has been known that the AKT pathway plays a key role in the regulation of cellular survival, apoptosis, and protein translation, and that it has prognostic significance in a number 
Table 1. Prognostic factors: clinicopathologic factors

\begin{tabular}{|c|c|c|c|c|c|c|c|}
\hline & \multirow{2}{*}{ No. } & \multicolumn{2}{|c|}{ 2-yr LRPFS } & \multicolumn{2}{|c|}{ 2-yr DMFS } & \multicolumn{2}{|c|}{$2-y r O S$} \\
\hline & & $\%$ & $p$-value & $\%$ & $p$-value & $\%$ & $p$-value \\
\hline Age (yr) & & & 0.970 & & 0.564 & & 0.779 \\
\hline$<65$ & 9 & 39 & & 56 & & 64 & \\
\hline$\geq 65$ & 12 & 35 & & 56 & & 47 & \\
\hline Gender & & & 0.726 & & 0.861 & & 0.980 \\
\hline Female & 9 & 30 & & 64 & & 58 & \\
\hline Male & 12 & 42 & & 54 & & 50 & \\
\hline ECOG PS & & & 0.497 & & 0.148 & & 0.209 \\
\hline 0 & 2 & 50 & & 100 & & 50 & \\
\hline 1 & 17 & 32 & & 46 & & 45 & \\
\hline 2 & 2 & 50 & & 100 & & 100 & \\
\hline Tumor location & & & 0.692 & & 0.841 & & 0.795 \\
\hline Hilar & 16 & 35 & & 55 & & 52 & \\
\hline Non-hilar & 5 & 40 & & 53 & & 60 & \\
\hline Nodal stage & & & 0.442 & & 0.821 & & 0.529 \\
\hline No & 13 & 39 & & 51 & & 60 & \\
\hline N1 & 8 & 31 & & 66 & & 44 & \\
\hline Preop CA19-9 (U/mL) & & & 0.916 & & 0.931 & & 0.620 \\
\hline$\leq 37$ & 7 & 43 & & 54 & & 42 & \\
\hline$>37$ & 14 & 35 & & 57 & & 57 & \\
\hline Type of surgery & & & 0.364 & & 0.232 & & 0.594 \\
\hline $\mathrm{R} 2$ resection & 19 & 35 & & 52 & & 55 & \\
\hline Bypass surgery & 2 & 50 & & 100 & & 50 & \\
\hline Chemotherapy & & & 0.275 & & 0.556 & & 0.863 \\
\hline No & 3 & 50 & & 33 & & 33 & \\
\hline Yes & 18 & 34 & & 59 & & 58 & \\
\hline RT course & & & 0.855 & & 0.549 & & 0.500 \\
\hline Split & 5 & 36 & & 67 & & 53 & \\
\hline Continuous & 16 & 40 & & 40 & & 60 & \\
\hline RT dose (Gy) & & & 0.709 & & 0.369 & & 0.603 \\
\hline$\leq 50.4$ & 11 & 44 & & 41 & & 51 & \\
\hline$>50.4$ & 10 & 27 & & 80 & & 57 & \\
\hline
\end{tabular}

LRPFS, locoregional progression-free survival; DMFS, distant metastasis-free survival; OS, overall survival; ECOG PS, the Eastern Cooperative Oncology Group performance status.

of cancers. The AKT pathway is activated by phosphorylation, so pAKT means activated AKT [15]. Some reports described AKT expression in bile duct cancers $[16,19,20]$. Chung et al. [15] reported PAKT expression was increased in $84.2 \%$ of 221 cases, and high pAKT group showed poor survival rate than low pAKT group $(p=0.06)$. In the study by Tanno et al. [19], 16 of 19 cases were positive for pAKT, and pAKT expression resulted in decreased radiosensitivity in the cell study. On the contrary, Javle et al. [20] suggested that high expression of AKT was correlated with improved survival in 24 cases of cholangiocarcinoma in multivariate analysis, and there was a significant association between AKT and pAKT in tumor tissues by Pearson correlation test.
CD24 is an adhesion receptor on activated endothelial cells and platelets and has been known to be associated with tumor proliferation, cell adhesion, motility, invasion, and apoptosis $[21,22]$. CD24 expression has been suggested as an adverse prognostic marker in several malignancies including cholangiocarcinoma. Agrawal et al. [23] studied 22 consecutive patients with cholangiocarcinoma, and the median survival of patients with high expression of CD24 was significantly shorter than those with low expression $(p=0.02)$. Among patients treated with chemotherapy, patients with low expression of CD24 had the median survival of 163 months, but it was only 17 months in patients with high expression $(p=0.04)$. This trend was sustained to patients treated with radiotherapy, 
Table 2. Prognostic factors: immunohistochemical results

\begin{tabular}{|c|c|c|c|c|c|c|c|}
\hline & \multirow{2}{*}{ No. } & \multicolumn{2}{|c|}{ 2-yr LRPFS } & \multicolumn{2}{|c|}{ 2-yr DMFS } & \multicolumn{2}{|c|}{$2-y r O S$} \\
\hline & & $\%$ & p-value & $\%$ & $p$-value & $\%$ & $p$-value \\
\hline pAKT cyt & & & 0.827 & & 0.535 & & 0.345 \\
\hline Negative & 11 & 36 & & 51 & & 64 & \\
\hline Positive & 10 & 42 & & 67 & & 43 & \\
\hline pAKT nuc & & & 0.564 & & 0.250 & & 0.989 \\
\hline Negative & 15 & 37 & & 48 & & 53 & \\
\hline Positive & 6 & 42 & & 83 & & 63 & \\
\hline CD24 & & & 0.408 & & 0.887 & & 0.610 \\
\hline Negative & 14 & 41 & & 57 & & 61 & \\
\hline Positive & 7 & 29 & & 54 & & 43 & \\
\hline MMPg & & & 0.817 & & 0.618 & & 0.478 \\
\hline Negative & 5 & 40 & & 53 & & 40 & \\
\hline Positive & 16 & 35 & & 55 & & 60 & \\
\hline Survivin & & & 0.502 & & 0.697 & & 0.611 \\
\hline Negative & 13 & 35 & & 53 & & 59 & \\
\hline Positive & 8 & 44 & & 63 & & 47 & \\
\hline Beta-catenin & & & 0.927 & & 0.161 & & 0.640 \\
\hline Negative & 18 & 30 & & 47 & & 52 & \\
\hline Positive & 3 & 33 & & 100 & & 67 & \\
\hline
\end{tabular}

LRPFS, locoregional progression-free survival; DMFS, distant metastasis-free survival; OS, overall survival; pAKT, phosphorylated protein kinase B; MMP9, matrix metalloproteinase 9.

Table 3. Summary of previous studies on chemoradiotherapy for gross residual disease after surgery

\begin{tabular}{lccccccc}
\hline & & & CCRT $^{\text {a) }}$ & & \multicolumn{3}{c}{ Median (mo) } \\
\cline { 5 - 7 } & & Year & No. & $(\%)$ & LRPFS & PFS & OS \\
\hline Ben-David et al. [32] & 2006 & $52^{\text {b) }}$ & 54 & NA & 7.9 & 13.1 \\
Oh et al. [33] & 2007 & 13 & 20 & NA & 13.0 & 10.0 \\
Present study & 2012 & 21 & 86 & & 15.5 & NA & 27.7 \\
\hline
\end{tabular}

CCRT, concurrent chemoradiotherapy; LRPFS, locoregional progression-free survival; PFS, progression-free survival; OS, overall survival; NA, not available.

a) The proportion of patients receiving CCRT is based on entire population of each study (R0, R1, and $\mathrm{R} 2$ resection). ${ }^{\text {b) Among }}$ 52 patients, there were 13 patients with distal extrahepatic bile duct (EHBD) cancer, 26 patients with hilar EHBD cancer and 13 patients with gallbladder cancer.

and the median survival of low expression of $C D 24$ versus high expression was 52 months versus 17 months $(p=0.08)$. In the study of 34 cases of resected cholangiocarcinoma by Keeratichamroen et al. [22], patients with high expression of CD24 had the median survival time of 9 months, whereas it was 24 months in patients with low expression $(p=0.007)$.

MMP9, a member of the matrix metalloproteinase, which digest the extracellular matrix required for cancer cell invasion
[24]. Shirabe et al. [25] analyzed 37 patients with surgically resected intrahepatic cholangiocarcinoma, and revealed that MMP9 expression was a prognostic factor associated with inferior survival. In contrast, Itatsu et al. [17] evaluated 66 cases of surgically resected EHBD cancers to find out the relationship between each subtype of MMPs and clinical outcomes. They proposed the expression of MMP7 was an unfavorable prognostic factor, but not for MMP9.

Survivin, an inhibitor of apoptosis protein, is not expressed in differentiated normal adult cells, but the overexpression was reported in bile duct cancers by several investigations [18,26-28]. Qin et al. [18] reported the significant difference of the mean survival time between patients with negative expression of survivin and positive expression (43.5 months for negative vs. 21.1 months for positive, $p<0.01$ ) in EHBD cancer. Javle et al. [28] also suggested survivin expression as a poor prognosticator in cholangiocarcinoma.

$\beta$-catenin has been known as an oncogene in many types of cancer, such as medulloblastoma, colon, thyroid, breast cancer and liver $[29,30]$. There was a study on the association between $\beta$-catenin and ampullary cancer, one of the periampullary cancers. In the study by Hsu et al. [31], loss of $\beta$-catenin expression was associated with disease recurrence on multivariate analysis. However, the prognostic value of $\beta$-catenin in EHBD 
cancer has not been reported.

In the present study, none of the 5 molecular biomarkers were correlated with the clinical outcomes. Given the retrospective nature of the study, the potential for selection bias cannot be excluded. Moreover, the tumor burden seems to be highly heterogeneous from the small gross residual disease to the bulky tumor, although the study population is relatively homogeneous in respect to the adjuvant treatment, that is, chemoradiotherapy. Also, small sample size might fail to detect the small significant difference.

In terms of clinical outcomes, the results of our study seem to be better than those of the previous studies (Table 3). However, patient population as well as tumor burden is highly heterogeneous among those studies including ours and moreover, the patient number is quite small. Further study with a larger cohort is needed for more understanding of this rare clinical situation.

In conclusion, it was impossible to find out prognostic value of pAKT, CD24, MMP9, survivin, and $\beta$-catenin in patients with EHBD cancer who received chemoradiotherapy after R2 resection or bypass surgery. Future research is needed on a larger data set or with other molecular biomarkers.

\section{Conflict of Interest}

No potential conflict of interest relevant to this article was reported.

\section{Acknowledgments}

This work was supported by KOSRO Young Investigator Fund.

\section{References}

1. Ganeshan D, Moron FE, Szklaruk J. Extrahepatic biliary cancer: new staging classification. World J Radiol 2012;4:345-52.

2. Seyama Y, Makuuchi M. Current surgical treatment for bile duct cancer. World J Gastroenterol 2007;13:1505-15.

3. Vauthey JN, Blumgart LH. Recent advances in the management of cholangiocarcinomas. Semin Liver Dis 1994;14:109-14.

4. Nathan $H$, Pawlik TM, Wolfgang $C L$, Choti MA, Cameron JL, Schulick RD. Trends in survival after surgery for cholangiocarcinoma: a 30-year population-based SEER database analysis. J Gastrointest Surg 2007;11:1488-96.

5. Witzigmann $H_{1}$ Berr $F$, Ringel $U$, et al. Surgical and palliative management and outcome in 184 patients with hilar cholangiocarcinoma: palliative photodynamic therapy plus stenting is comparable to $\mathrm{r} 1 / \mathrm{r} 2$ resection. Ann Surg 2006;244:230-9.

6. Reding R, Buard JL, Lebeau G, Launois B. Surgical management of 552 carcinomas of the extrahepatic bile ducts (gallbladder and periampullary tumors excluded): results of the French Surgical Association Survey. Ann Surg 1991;213:236-41.

7. Yamaguchi K, Chijiiwa K, Saiki S, Shimizu S, Takashima M, Tanaka M. Carcinoma of the extrahepatic bile duct: mode of spread and its prognostic implications. Hepatogastroenterology 1997;44:1256-61.

8. Nagorney DM, Donohue JH, Farnell MB, Schleck CD, Ilstrup DM. Outcomes after curative resections of cholangiocarcinoma. Arch Surg 1993;128:871-7.

9. Kim JW, Jo S, Moon HJ, et al. Prognostic factors after major resection for distal extrahepatic cholangiocarcinoma. Korean J Gastroenterol 2006;47:144-52.

10. Kloek JJ, Ten Kate FJ, Busch OR, Gouma DJ, van Gulik TM. Surgery for extrahepatic cholangiocarcinoma: predictors of survival. HPB (Oxford) 2008;10:190-5.

11. Fingas $C D$, Katsounas $A$, Kahraman $A$, et al. Prognostic assessment of three single-nucleotide polymorphisms (GNB3 825C>T, BCL2-938C>A, MCL1-386C>G) in extrahepatic cholangiocarcinoma. Cancer Invest 2010;28:472-8.

12. Mobius $C$, Demuth $C$, Aigner $T$, et al. Evaluation of VEGF $A$ expression and microvascular density as prognostic factors in extrahepatic cholangiocarcinoma. Eur J Surg Oncol 2007;33: 1025-9.

13. Tamada S, Shibahara $H$, Higashi $M$, et al. MUC4 is a novel prognostic factor of extrahepatic bile duct carcinoma. Clin Cancer Res 2006;12:4257-64.

14. Li S, Jo YS, Lee JH, et al. L1 cell adhesion molecule is a novel independent poor prognostic factor of extrahepatic cholangiocarcinoma. Clin Cancer Res 2009;15:7345-51.

15. Chung JY, Hong SM, Choi BY, Cho H, Yu E, Hewitt SM. The expression of phospho-AKT, phospho-mTOR, and PTEN in extrahepatic cholangiocarcinoma. Clin Cancer Res 2009;15: 660-7.

16. Hori $H$, Ajiki T, Mita $Y$, et al. Frequent activation of mitogenactivated protein kinase relative to Akt in extrahepatic biliary tract cancer. J Gastroenterol 2007;42:567-72.

17. Itatsu K, Zen Y, Yamaguchi J, et al. Expression of matrix metalloproteinase 7 is an unfavorable postoperative prognostic factor in cholangiocarcinoma of the perihilar, hilar, and extrahepatic bile ducts. Hum Pathol 2008;39:710-9.

18. Qin XL, Xue HZ, Wang ZR, Liu HS, Zhou HB, Ma W. Expression of Survivin protein in extrahepatic cholangiocarcinoma and its relationship with the prognosis. Zhonghua Wai Ke Za Zhi 2009;47:1852-6.

19. Tanno S, Yanagawa N, Habiro A, et al. Serine/threonine kinase AKT is frequently activated in human bile duct cancer and is associated with increased radioresistance. Cancer Res 
2004;64:3486-90.

20. Javle MM, Yu J, Khoury $T$, et al. Akt expression may predict favorable prognosis in cholangiocarcinoma. J Gastroenterol Hepatol 2006;21:1744-51.

21. Kristiansen G, Sammar M, Altevogt P. Tumour biological aspects of CD24, a mucin-like adhesion molecule. J Mol Histol 2004;35:255-62.

22. Keeratichamroen $S$, Leelawat $K_{1}$ Thongtawee $T$, et al. Expression of CD24 in cholangiocarcinoma cells is associated with disease progression and reduced patient survival. Int J Oncol 2011; 39:873-81.

23. Agrawal $S$, Kuvshinoff BW, Khoury $T$, et al. CD24 expression is an independent prognostic marker in cholangiocarcinoma. J Gastrointest Surg 2007;11:445-51.

24. Coussens LM, Fingleton B, Matrisian LM. Matrix metalloproteinase inhibitors and cancer: trials and tribulations. Science 2002;295:2387-92.

25. Shirabe $K_{1}$ Shimada $M$, Kajiyama $K_{1}$ et al. Expression of matrix metalloproteinase- 9 in surgically resected intrahepatic cholangiocarcinoma. Surgery 1999;126:842-6.

26. Ambrosini G, Adida C, Altieri DC. A novel anti-apoptosis gene, survivin, expressed in cancer and lymphoma. Nat Med 1997;3:917-21.

27. Chang Q, Liu ZR, Wang DY, Kumar M, Chen YB, Qin RY. Survivin expression induced by doxorubicin in cholangiocarcinoma. World J Gastroenterol 2004;10:415-8.

28. Javle MM, Tan D, Yu J, et al. Nuclear survivin expression predicts poor outcome in cholangiocarcinoma. Hepatogastroenterology 2004;51:1653-7.

29. Thompson MD, Monga SP. WNT/beta-catenin signaling in liver health and disease. Hepatology 2007;45:1298-305.

30. Nault JC, Zucman-Rossi J. Genetics of hepatobiliary carcinogenesis. Semin Liver Dis 2011;31:173-87.

31. Hsu HP, Shan YS, Jin YT, Lai MD, Lin PW. Loss of E-cadherin and beta-catenin is correlated with poor prognosis of ampuIlary neoplasms. J Surg Oncol 2010;101:356-62.

32. Ben-David MA, Griffith KA, Abu-Isa E, et al. External-beam radiotherapy for localized extrahepatic cholangiocarcinoma. Int J Radiat Oncol Biol Phys 2006;66:772-9.

33. Oh D, Lim do $H_{1}$ Heo JS, et al. The role of adjuvant radiotherapy in microscopic tumor control after extrahepatic bile duct cancer surgery. Am J Clin Oncol 2007;30:21-5. 